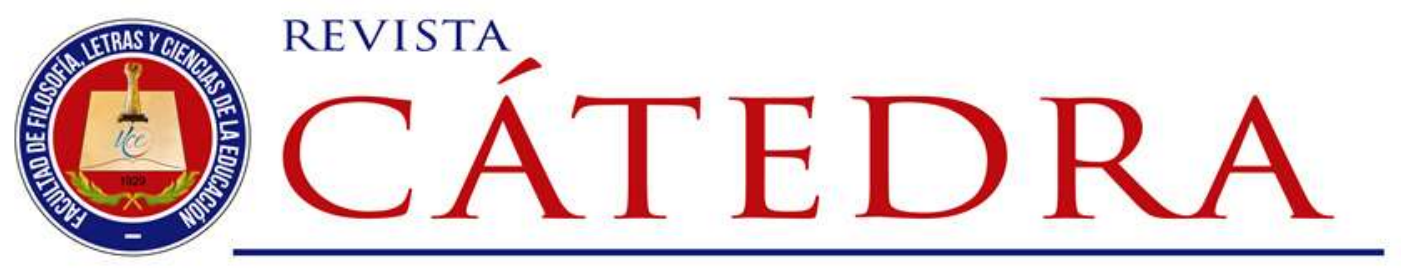

\title{
Autorregulación del aprendizaje en estudiantes universitarios: un estudio descriptivo
}

\section{Self-regulation of learning in university students: $a$ descriptive study}

\author{
Pablo Burbano-Larrea \\ Universidad Central del Ecuador, Quito, Ecuador \\ pdburbano@uce.edu.ec \\ https://orcid.org/0000-0002-4476-9407 \\ Mirian Basantes-Vásquez \\ Universidad Central del Ecuador, Quito, Ecuador \\ msbasantes@uce.edu.ec \\ https://orcid.org/0000-0001-5960-312X \\ Isabel Ruiz-Lapuerta \\ Universidad Central del Ecuador, Quito, Ecuador \\ ipruiz@uce.edu.ec \\ https://orcid.org/0000-0002-0226-729X
}

(Recibido: 09/06/2021; Aceptado: 15/06/2021/ Versión final recibida: 15/08/2021)

Cita del artículo: Burbano-Larrea, P., Basantes-Vásquez, M. y Ruiz-Lapuerta, I. (2021). Autorregulación de los Aprendizajes en estudiantes universitarios: un estudio descriptivo. Revista Cátedra, 4(3), 74-92.

\section{Resumen}

Entre las capacidades fundamentales con las que debe contar el ser humano para alcanzar un desarrollo exitoso a nivel personal, académico y profesional se encuentra la autorregulación del aprendizaje. Gestionar los recursos y estrategias en los procesos de construcción del conocimiento resulta indispensable. Solo de esa manera se puede planificar, ejecutar, monitorear y evaluar los desempeños y los resultados formativos orientados a las metas. El objetivo del presente trabajo fue determinar la autorregulación del aprendizaje en los estudiantes de la Facultad de Filosofía, Letras y Ciencias de la Educación, de la Universidad Central del Ecuador. Esta investigación se desarrolló desde un

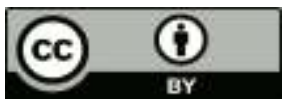


enfoque cuantitativo y un nivel descriptivo, siendo un estudio de tipo trasversal. Los hallazgos encontrados permitieron concluir que dicho grupo de investigación presentan en su mayoría niveles bajos y medio bajos de autorregulación general. La autorregulación del aprendizaje presenta los siguientes factores: planificación, gestión de la cognición, gestión de la motivación, evaluación de la comprensión y gestión del contexto. En cada uno de ellos se pudo identificar niveles: bajos y medios bajos entre los estudiantes. Se encontraron resultados similares en el análisis por sexo y por nivel de formación. Al contrastar estos resultados con otros estudios se observó coincidencias y contradicciones. Lo cual señala la necesidad de profundizar la investigación de esta variable. Se sugiere generar innovaciones curriculares en la educación universitaria que permitan el desarrollo de la autorregulación de los aprendizajes a lo largo de su formación profesional.

\section{Palabras clave}

Autorregulación del aprendizaje, gestión de la cognición, gestión del contexto, estudiantes universitarios, evaluación de la comprensión, planificación, gestión de la motivación.

\section{Summary}

Among the fundamental capacities that the human being must have to achieve successful development at a personal, academic and professional level is the self-regulation of learning. Managing resources and strategies in knowledge construction processes is essential. Only in this way can goal-oriented performance and training results be planned, executed, monitored and evaluated. Within this order of ideas, the objective of this work was to determine the self-regulation of learning in students of the Faculty of Philosophy, Letters and Education Sciences, of the Central University of Ecuador. It was developed from a quantitative approach and a descriptive level, being a cross-sectional study. The discoveries found allowed us to conclude that those research group mostly present low and medium-low levels of self-regulation, with similar results in the analysis by sex and by level of training. Likewise, it was possible to identify that the levels: low and medium low are prevalent among students in the various factors of this variable. Being these: planning, cognition management, motivation management, understanding assessment and context management. When contrasting these results with other studies, coincidences and contradictions were observed. This indicates the need to deepen the investigation of this variable. It is suggested to generate curricular innovations in university education that allow the development of self-regulation of learning throughout their professional training.

\section{Keywords}

Self-regulation of learning, cognitive management, context management, university students, comprehension assessment, planning, motivation management.

\section{Introducción}

Sin duda en todo proceso académico el principal protagonista es y debe ser el estudiante. La construcción y adquisición de saberes por parte del sujeto que aprende son la razón de ser de la educación. De allí la importancia de estudiar las variables que intervienen en la consecución de las metas de aprendizaje. En la educación superior o universitaria el interés investigativo se enfoca en los mismos aspectos. Aún más, considerando la trascendencia de alcanzar un aprendizaje sólido que se reflejará en el ejercicio profesional. "los actuales desafíos demandados a la formación en educación superior exigen situar el foco en la evidencia de logro de aprendizaje en estudiantes" (García-Gajardo, Fonseca-Grandón y Concha-Gfell, 2015, p. 2). 
Rosário y sus colaboradores (2014, pp. 782-783) señalan que no se puede atribuir a la inteligencia las diferencias en los resultados de aprendizaje de los estudiantes. Se identifican factores cognitivos, motivacionales y conductuales. Está claro cada vez más que el estudiante tiene un rol fundamental en su propio proceso de aprendizaje. El estudio de la autorregulación en relación con otras variables es fundamental para comprender el fenómeno del aprendizaje. En ese contexto y como uno de los elementos claves que participan en el proceso educativo del estudiante se encuentra la autorregulación de los aprendizajes.

Este constructo se define como el conjunto de estrategias que posee el sujeto que le permiten planificar, monitorear y evaluar su proceso de aprendizaje. El presente estudio tiene como objetivo establecer la autorregulación del aprendizaje en estudiantes de educación superior.

El desempeño académico y la consolidación de los aprendizajes de los estudiantes en formación docente, es una constante preocupación de las instituciones de educación superior. Lo cual motiva la investigación de las dimensiones de la autorregulación del aprendizaje, y describir los niveles con los que cuentan los estudiantes en cada una de ellas.

La teoría señala que la autorregulación del aprendizaje se constituye en un importante mecanismo que permite anticipar el éxito académico entre los estudiantes. Según mencionan Hernández-Barrios y Camargo-Uribe (2017) "su relevancia radica en que constituye una de las mejores variables predictoras del rendimiento académico" (p. 147). Los estudiantes que tienen altos niveles en el uso de diversas estrategias de autorregulación obtienen mejores resultados. Gaeta-González (2006) señalan que "el que los alumnos sean participes activos de su propio aprendizaje y puedan llegar a aprender de forma autónoma y autorregulada se considera como un aspecto fundamental del óptimo aprendizaje" (p. 2).

Otra importante motivación tanto de autoridades como docentes es ofrecer una formación integral que dote a los futuros profesionales no solo de conocimiento sino de múltiples competencias. Es importante considerar "las exigencias del contexto nacional e internacional de formar recursos humanos que se encuentren preparados para enfrentar las nuevas necesidades del mercado laboral y el contexto globalizado y multicultural de nuestras sociedades, implican una educación integral" (Luy-Montejo, 2019, p. 354). Entre ellas las capacidades de gestionar sus procesos de aprendizaje será clave en el éxito laboral, en un entorno que exige estar permanentemente actualizado. El contar con autorregulación de los aprendizajes es evidencia de disciplina y control de la conducta y la cognición, esto es característico de profesionales responsables y comprometidos.

Abordar este tema de investigación permite enriquecer el conocimiento científico basado en evidencias en estudiantes universitarios. Identificar ¿Qué nivel de autorregulación del aprendizaje tienen los estudiantes? ¿Existen diferencias en esta variable entre hombres y mujeres? ¿Qué niveles presentan los cinco factores de autorregulación del aprendizaje? permitirá orientar esfuerzos acciones de fortalecimiento en los planes de formación de los futuros docentes. Como lo manifiesta Hernández-Barrios y Camargo-Uribe (2017) "esta información es de central importancia al momento de diseñar e implementar planes, programas y acciones en los escenarios formativos que contribuyan al avance de los estudiantes en sus estudios académicos" (p. 156).

Existe escaza información sobre la autorregulación en educación superior en el contexto ecuatoriano. La Pandemia causada por el COVID-19, con el consecuente confinamiento y la falta de instrumentos adaptados al contexto ecuatoriano, se identifican como obstáculos en 
este estudio. Dichos desafíos fueron superados con el apoyo institucional y de los participantes.

El presente artículo está estructurado de la siguiente manera, en la sección 2, la revisión de la literatura; es decir los contenidos relacionados con la autorregulación del aprendizaje. La sección 3 se refiere a los métodos y materiales utilizados en la presente investigación, además se contrastó con investigaciones realizadas por otros autores. En la sección 4 se detallan los hallazgos encontrados después de la aplicación del instrumento respectivo. En la sección 5 se detalla la discusión de resultados y en la sección 6 se describen las conclusiones pertinentes.

\section{Revisión de la literatura}

\subsection{El aprendizaje}

El aprendizaje ha sido definido desde diferentes teorías, así por ejemplo desde el Conductismo "se entiende al aprendizaje como aquellos cambios que se producen en la conducta de las personas en respuesta al ambiente" (Prados, Reyna y Rey, 2014, p. 21). Así desde el constructivismo social, se le considera como "la internalización de conocimientos o habilidades por medio de la experiencia, el ejercicio o el estudio, y constituye un proceso esencial para integrarnos en la sociedad desde los primeros años de vida" (Catuara, 2018, p. 73).

El aprendizaje es un proceso permanente en el ser humano, no se desarrolla únicamente en la infancia, sino que está presente en todo el ciclo de vida. En el transcurrir evolutivo, la persona realiza cambios importantes que le permite integrarse al contexto en donde se desenvuelve.

\subsection{Autorregulación}

La autorregulación es la capacidad de un individuo para controlar y manejar su propia conducta. Es así como "implica la modulación del pensamiento, la motivación, la atención y la conducta, mediante la utilización deliberada o automatizada de mecanismos específicos y de estrategias de apoyo" (González, 2001, p. 1). Por lo que le posibilita a la persona guiar su actuación orientada hacia las metas, a lo largo del tiempo y en diversos contextos.

La persona, en cada momento de su vida planifica, ejecuta y evalúa acciones que le ayudan a cumplir determinados objetivos de una manera eficiente. A este proceso se le conoce con el nombre de autorregulación.

La autorregulación es, según Panadero y Alonso-Tapia (2014) "el control que una persona ejecuta sobre sus pensamientos, acciones, emociones y motivación mediante estrategias personales para alcanzar un objetivo de aprendizaje establecido" (p. 451). Es decir que todos los seres humanos pueden realizar acciones en el plano cognitivo, actitudinal y comportamental para alcanzar los objetivos que se hayan propuesto.

\subsection{Autorregulación del aprendizaje}

La acción educativa asume la educabilidad del discente, su enseñanza y guía hacia la adquisición de habilidades y competencias que garanticen su adecuado desarrollo, autorrealización personal y profesional. Al respecto López (1992), indica "es importante proporcionar al estudiante estrategias y métodos que desarrollen en él autorregulación y autocontrol" (p. 111). El desarrollo de tales capacidades brinda coherencia entre la conducta y el pensamiento; es decir, la persona dispone de las facultades que permiten 
reflexionar su accionar, y en caso de requerir mejora esta posee las acciones necesarias para corregirla.

El término autorregulación del aprendizaje data de "principios de la década de los sesenta" (González, 2001, p. 4). Emerge a través de cuestionamientos sobre la adquisición, apropiación del conocimiento, y la autonomía que poseen los estudiantes en su proceso de aprendizaje. Según Kauffman et. al (2006), tiene sus "inicios desde la psicología cognitiva, cuyo origen se remonta a la teoría del aprendizaje cognitivo social propuesto por Bandura, asigna relevancia al Determinismo Recíproco" sugiriendo que el aprendizaje es el resultado de factores personales, ambientales y de comportamiento (p. 1063).

Kaufmann et. al (2006) señala "en 1980 Albert Bandura, en su Teoría del Aprendizaje Social, introduce el concepto de aprendizaje autorregulado, que es aplicado en todas las actividades del ser humano, pero específicamente en el contexto educativo" (p. 1063). El autor apoya la postura de Bandura y considera que la autorregulación es un proceso que tiene influencia social. Es a partir de varias investigaciones, que surgen las concepciones actuales de la autorregulación en el aprendizaje.

González señala que el interés inicial de Bandura se centró en el autocontrol, entendido como la capacidad para ejercer su dominio sobre las propias acciones en ausencia de limitaciones externas inmediatas. Más adelante el autor introdujo el término autorregulación. Este implicaba el establecimiento de una meta previa, la presencia de la autoevaluación y la administración de autorrefuerzo, además de la ejecución de la respuesta aprendida. Finalmente resaltó el papel de la autoeficacia definida como la autoconvicción de que se puede ejecutar con éxito la conducta requerida para producir ciertos resultados. (González, 2011, pp. 3-4).

Zimmerman y Martínez-Pons (1998) expresaron que la autorregulación del aprendizaje deriva de la actividad metacognitiva, motivada y conductualmente activa de los estudiantes como participantes de sus propios procesos de aprendizaje. Estos autores señalaron que:

En términos de los procesos metacognoscitivos, los aprendices autorregulados planifican y organizan sus actividades de aprendizaje y se autoevalúan durante este proceso. Desde el punto de vista motivacional, se perciben a sí mismos como autoeficaces, autónomos y motivados intrínsecamente. Desde la perspectiva de su comportamiento, seleccionan, estructuran y crean ambientes sociales y físicos que potencian su proceso de aprendizaje. (p. 284).

Es decir, los estudiantes autorregulados toman decisiones y utilizan los métodos necesarios para alcanzar los objetivos de aprendizaje que se establecen. Los estudiantes al encontrarse motivados son eficaces en el momento de cumplir con una tarea hasta alcanzar las metas que se han planteado.

Desde una perspectiva amplia, la autorregulación es el proceso que permite desarrollar estrategias para el cumplimiento de objetivos o tareas, de hecho, Zimmerman (2008), considera que "es un proceso proactivo que los estudiantes utilizan para adquirir habilidades académicas, como establecer metas, seleccionar e implementar estrategias y autocontrolar la efectividad de uno mismo" (p. 166). Por lo que se afirma que el educando desarrolla procesos cognitivos para conseguir su éxito académico.

Castro-Pereira señala que la capacidad de autorregulación no es innata, debe ser desarrollada. Se ha de facultar al sujeto de medios que permitan la identificación de la madurez, la motivación, los factores psico-sociales o procesos intrapersonales e 
interpersonales que influyen en su conducta. (Castro-Pereira, 1989, pp. 92-93). Un estudiante autorregulado que participa en su propio aprendizaje se considera un estudiante competente.

Ciertamente, el sujeto de educación se encuentra en una constante interacción con el contexto e información que le rodea. De ahí que, Pintrich supone que:

Learners are assumed to actively construct their own meanings, goals, and strategies from the information available in the external environment as well as information in their own minds (the internal environment). Learners are not just passive recipients of information from teachers, parents, or other adults, but rather active, constructive meaning makers as they go about learning. [Los alumnos construyen activamente sus propios significados, objetivos y estrategias a partir de la información disponible en el entorno externo, así como de la información en sus propias mentes (el entorno interno). Los estudiantes no son sólo pasivos receptores de información de maestros, padres u otros adultos, sino más bien creadores de significados activos y constructivos a medida que avanzan en el aprendizaje.] (Pintrich, 2000, p. 452).

De modo que, la autorregulación del aprendizaje es un proceso activo, en el cual "los sujetos establecen los objetivos que guían su aprendizaje intentando monitorizar, regular y controlar su cognición, motivación y comportamiento con la intención de alcanzarlos". (Rosário, et al, 2010, p. 829). Biggs señala que los alumnos aprenden con mayor facilidad cuando son influenciados por lo que ellos quieren aprender. Los motivos para realizar una tarea concreta de aprendizaje determinan las estrategias a utilizar, y éstas los resultados del aprendizaje (Biggs, 1993, pp. 5-8).

Al respecto Boekaerts y Corno refieren que "there are no direct linkages between achievement and personal or contextual characteristics; achievement effects are mediated by the self-regulatory activities that students engage to reach learning and performance goals". [No existen vínculos directos entre el logro y las características personales o contextuales; los efectos del rendimiento están mediados por las actividades de autorregulación que los estudiantes realizan para alcanzar las metas de aprendizaje y desempeño] (Boekaerts y Corno, 2005, p. 201). De modo que los educandos procuran completar una tarea planificando qué estrategias van a utilizar.

No obstante, el estudiante no es un nato regulador de su aprendizaje o experto en estrategias por lo que su éxito académico se podría ver frustrado. "Lo que identifica como autorregulador de su aprendizaje a un individuo no es tanto la utilización aislada de estrategias de aprendizaje, sino su iniciativa personal, su perseverancia en la tarea y las competencias exhibidas, independientemente del contexto de aprendizaje." (Bandura, et al, 2001, p. 187).

El aprendizaje académico autorregulado surge, según Zimmerman (2001) a mediados de los ochenta. Con el propósito de responder a la cuestión de cómo los estudiantes llegan a dominar sus propios procesos de aprendizaje. La autorregulación del aprendizaje se refiere al "proceso auto-directivo por el cual los estudiantes transforman sus habilidades mentales en destrezas académicas relacionadas con las tareas de aprendizaje" (p. 12).

Zimmerman y Schunk se refieren al proceso por el cual los estudiantes se activan personalmente y sostienen cogniciones, afectos y conductas que son sistemáticamente orientadas hacia el logro de objetivos de aprendizaje (Zimmerman y Schunk, 2011, p. 11). 
Al establecer objetivos de aprendizaje, los estudiantes crean un proceso cíclico de autoorientación por el cual monitorean la efectividad de las estrategias y adaptan su trabajo de acuerdo con sus resultados.

Para propósitos de esta investigación se entiende a la autorregulación del aprendizaje como un proceso cognitivo. Este facilita la adquisición de conocimientos por medio de la aplicación intencional de estrategias metacognitivas, que tienen como propósito el formar estudiantes autónomos y motivados en su proceso de aprendizaje.

Panadero y Alonso-Tapia recalcan la importancia de las fases de la autorregulación del aprendizaje propuesto por Zimmerman. Siendo las mismas la planificación, ejecución y la autorreflexión. (Panadero y Alonso-Tapia, 2014, pp. 451-452).

Zimmerman y Schunk señalan que el aprendizaje autorregulado es un medio efectivo para mejorar el desempeño estudiantil, además que los estudiantes se autorregulan en procesos que consolidan su aprendizaje de manera efectiva. Así, se asume que los estudiantes:

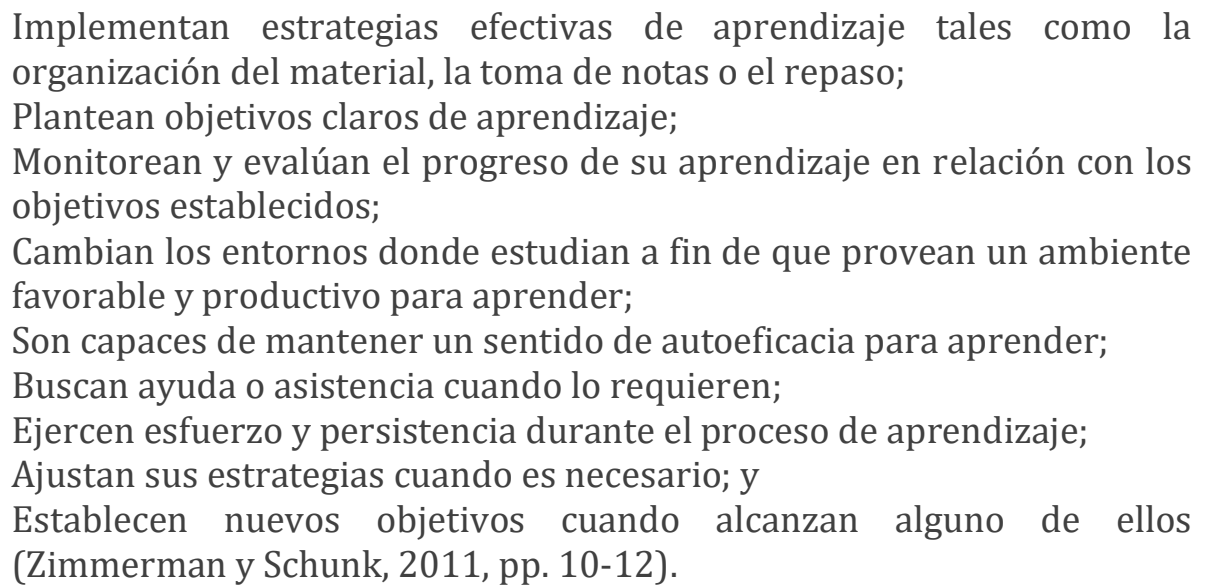

En esta revisión se puede apreciar que un aspecto crítico de la autorregulación del aprendizaje es que los estudiantes tengan opciones disponibles para tomar decisiones sobre la tarea. Las tareas requieren que parte de sus componentes sean regulados externamente (por el profesor u otro estudiante más avanzado) y otros por ellos mismos. Del mismo modo, Pintrich postula una perspectiva socio-cognoscitiva que propone una estructura de la autorregulación del aprendizaje, que sistematiza en cuatro fases (planificación, supervisión, revisión y valoración) y, al mismo tiempo, en cinco áreas (cognitiva, afectivo motivacional, comportamental y contextual). Ayudando a la integración de los subprocesos implicados en la autorregulación que se interrelacionan unos con otros y se ejecutan diferencialmente en función de los cuatro momentos del proceso de aprendizaje (Pintrich, 2000, pp. 451-453). Las fases mencionadas, representan para Pintrich una secuencia general, pero según Cruz, et al. (2017), afirma que "no necesariamente se presentan en ese orden, sino que tales fases pueden manifestarse de manera dinámica o simultánea" (p. 1).

Los diferentes modelos de autorregulación del aprendizaje proponen distintas herramientas de evaluación sobre la autorregulación del aprendizaje, uno de ellos el ARATEX-R. El cual pretende determinar las "estrategias generales de autorregulación, el cuestionario está centrado en la lectura de textos, una de las actividad es más frecuentes en la vida de los alumnos universitarios." (Nuñez et. al, 2015, p. 10). El instrumento abarca factores de primer orden; encargados de abordar lo intrínseco y extrínseco de la persona. 
El ARATEX-R presenta cinco factores de autorregulación del aprendizaje: 1) gestión de la planificación, 2) gestión de la cognición, 3) gestión de la motivación, 4) evaluación de la comprensión y 5) gestión del contexto. Si bien existen diferentes modelos para explicar y conceptualizar este proceso, todos tienen rasgos y características similares, los factores presentados a continuación guardan lo sustancial de los postulados expresados por Pintrich y Zimmerman.

El primer factor gestión de la planificación, según Pintrich, "establece los objetivos, así como la activación de las percepciones y el conocimiento de la tarea y el contexto del yo en relación con la tarea" (Pintrich, 2000, p. 3). El segundo factor gestión de la cognición o seguimiento se refiere a varios "procesos de seguimiento que representan la conciencia metacognitiva de diferentes aspectos del yo o la tarea y el contexto" (Pintrich, 2000, p.3). Es decir, este factor busca que los procesos cognitivos superiores se dispongan en funcionamiento para un mejor resultado en la ejecución de la tarea.

El tercer factor gestión de la motivación o control - regulación según Pintrich alude que el sujeto debe implicar esfuerzos para controlar y regular diferentes aspectos del yo o la tarea y el contexto. Por su parte Zimmerman incluye al factor motivacional dentro de la etapa de planificación. Si bien este factor se encuentra en distintas etapas los autores coinciden en la importancia de la motivación del sujeto para el alcance de los objetivos propuestos al inicio de la tarea.

Finalmente, los factores cuatro y cinco denominados: evaluación de la comprensión y gestión del contexto se encuentran inmersos en la etapa de reacción y reflexión según lo expuesto por Pintrich y autorreflexión postulada por Zimmerman. Estás tratan de los "varios tipos de reacciones y reflexiones sobre el yo y la tarea o contexto." (Pintrich, 2000, p.3) en estas el aprendiz debe analizar los resultados obtenidos y las posibles mejoras que puede realizar a futuro en caso de no alcanzar su objetivo inicial.

\section{Métodos y materiales}

Este estudio se encuadró en un enfoque cuantitativo de investigación, considerando que tanto el instrumento de recolección de datos ARATEX-R presenta los resultados en valores numéricos. Así como el procesamiento y análisis de dichos datos se realizó mediante procedimientos estadísticos. La técnica de muestreo pretendió generalizar los resultados a toda la población de los estudiantes de la Facultad de Filosofía, Letras y Ciencias de la Educación de la Universidad Central del Ecuador.

El nivel de esta investigación correspondió al descriptivo, al presentar el nivel general de autorregulación del aprendizaje, los niveles en cada uno de los cinco factores parte de esta variable, así como la distribución por sexo y por unidad de formación. Los resultados se presentaron mediante frecuencias y porcentajes, comparándolos con los hallazgos de otros estudios. El estudio fue de tipo trasversal, en este caso la aplicación del instrumento se ejecutó entre los meses de junio y julio del 2020.

La población de estudio correspondió a 4199 estudiantes de la modalidad presencial legalmente matriculados entre primer y noveno semestre de diez carreras de la Facultad de Filosofía de la Universidad Central del Ecuador. Mediante muestreo probabilístico aleatorio simple, otorgando la posibilidad de participar a todos los elementos de la población, disminuyendo la intervención de variables extrañas que pudieran incidir en los resultados.

Se aplicó la formula correspondiente y la ejecución de las operaciones señaladas en la ecuación 1 , con un $5 \%$ de error y $95 \%$ de confiabilidad, se obtuvo la necesidad de contar con 352 participantes. Considerando la situación de cuarentena producido por la crisis 
sanitaria ocasionada por el Covd-19, se realizó la aplicación del instrumento de manera virtual, lo que produjo un importante incremento en el número final de participantes. Tras la depuración de los datos y un análisis de estratificación, para mantener constante la distribución de resultados por carrera, sexo y unidad de formación. Finalmente se trabajó con una muestra participante de 1576 estudiantes, que accedieron a dar el correspondiente asentimiento informado.

$$
\frac{k^{2} \cdot p \cdot q \cdot N}{\left(e^{2}(N-1)\right)+k^{2} \cdot p \cdot q}
$$

Donde:

K: Corresponde al nivel de confianza 95\%, 1.96

p: es la proporción de individuos que poseen en la población la característica de estudio, 0.5

q: s la proporción de individuos que no poseen esa característica, es decir, es 1-p, 0.5

e: el error aceptable $5 \%$

N: es el tamaño de la población o universo, 4199

La técnica usada fue la encuesta mediante la aplicación del cuestionario ARATEX-R, este instrumento presenta 20 ítems que permiten establecer 5 factores de autorregulación del aprendizaje y un puntaje general de dicha variable. El instrumento se rediseñó de manera digital y se realizó su aplicación mediante la herramienta Google forms, enviando el enlace a los correos electrónicos institucionales de los estudiantes. Para establecer la confiabilidad de este instrumento se realizó un estudio piloto a cuyos resultados se les aplicó el estadístico alfa de Cronbach de donde se obtuvo un puntaje de 0.931 el mismo que indica alta confiabilidad.

Se tabuló los resultados obtenidos de manera automática en la misma herramienta informática y se procedió a exportar los mismos a una hoja de cálculo de Excel. Tras una primera depuración, finalmente se realizó los análisis estadísticos requeridos en el SPSS.

Considerando que el coeficiente de asimetría donde los valores en todos los casos se encuentran inferiores y distantes de cero. Y las diferencias entre la media, moda y mediana son mayores, la distribución no se ajusta a la normalidad. En consecuencia, no se pudo realizar una categorización de los puntajes mediante valores $\mathrm{z}$, generando puntos de corte en base a desviaciones típicas.

Se procedió a transformar los puntajes directos en percentiles, se determinó cinco puntos de corte en la distribución de cada uno de los factores. Se estableció los niveles: bajo, medio bajo, medio, medio alto y alto, en cuatro de los factores de la variable (planificación, gestión de la cognición, gestión de la motivación y evaluación de la comprensión), así como en el puntaje general de la variable. En el factor gestión del contexto por las características de la distribución de resultados solo fue posible generar tres niveles: bajo, medio y alto.

\section{Resultados}

Sobre la autorregulación del aprendizaje de manera general. Se encontró que el $27.0 \%$ equivalente a 425 estudiantes se ubican en el nivel bajo. El $20.4 \%$ es decir 321 estudiantes en el nivel medio bajo. $29.8 \%$ correspondientes a 470 estudiantes en el nivel medio. El 14.8 $\%$ es decir 233 estudiantes en el nivel medio alto. Y apenas el $8.1 \%$ ósea 127 estudiantes se 
ubican en el nivel alto. Los estudiantes en su mayoría no cuentan con el conjunto de estrategias adecuadamente desarrolladas que les permita planificar, monitorear y evaluar sus procesos de formación para la consecución de metas académicas. Evidenciando limitaciones en la motivación en el logro de tareas y manejo de los elementos del contexto de aprendizaje.

Los resultados en los niveles de autorregulación del aprendizaje por sexo. Se encontró entre las mujeres que el $26.7 \%$ equivalente a 278 estudiantes se ubican en el nivel bajo. El 20.6 $\%$ es decir 214 estudiantes en el nivel medio bajo. $29.7 \%$ correspondientes a 309 estudiantes en el nivel medio. El $14.8 \%$ es decir 154 estudiantes en el nivel medio alto. Y apenas el $8.3 \%$ ósea 86 estudiantes se ubican en el nivel alto. Dando un total de 1041 mujeres.

En los hombres se hallo que el $27.5 \%$ equivalente a 147 estudiantes se ubican en el nivel bajo. El $20 \%$ es decir 107 estudiantes en el nivel medio bajo. $30.1 \%$ correspondientes a 161 estudiantes en el nivel medio. El $14.8 \%$ es decir 79 estudiantes en el nivel medio alto. Y apenas el $7.7 \%$ ósea 41 estudiantes se ubican en el nivel alto. Dando un total de 535 hombres. No existe diferencia en los resultados de hombres y mujeres entre los estudiantes participantes con relación a los resultados generales de grupo.

El Cuadro 1 presenta los resultados de autorregulación del aprendizaje por unidades de formación de las carreras de educación. La unidad básica está conformada por estudiantes entre primer y tercer semestre, donde un $46 \%$ de los mismos presentan niveles bajos o medio bajos. La unidad profesional está integrada por estudiantes entre cuarto y octavo semestre, en la cual un $48 \%$ de participantes se ubican entre los niveles: bajos y medios bajos. Los estudiantes de noveno semestre forman parte de la unidad de titulación, donde el $49 \%$ se ubican en los niveles: bajo y medio bajo. Estos hallazgos muestran coincidencia en que la mayoría de la muestra se ubica en este nivel, independientemente de la unidad de formación a la que formen parte.

\begin{tabular}{ccccccc}
\hline NIVEL & \multicolumn{2}{c}{ BASICO } & \multicolumn{2}{c}{ PROFESIONAL } & \multicolumn{2}{c}{ TITULACIÓN } \\
\hline & $\mathbf{f i}$ & $\mathbf{h i \%}$ & $\mathbf{f i}$ & $\mathbf{h i \%}$ & $\mathbf{f i}$ & $\mathbf{h i \%}$ \\
Bajo & 155 & 25 & 212 & 28 & 58 & 30 \\
Medio & 128 & 21 & 157 & 20 & 36 & 19 \\
bajo & & 32 & 227 & 30 & 46 & 24 \\
Medio & 197 & 14 & 115 & 15 & 29 & 15 \\
Medio & 89 & 76 & 7 & 25 & 13 \\
alto & 46 & 7 & 56 & $\mathbf{1 0 0}$ & $\mathbf{1 9 4}$ & $\mathbf{1 0 0}$ \\
Alto & $\mathbf{6 1 5}$ & $\mathbf{1 0 0}$ & $\mathbf{7 6 7}$ & & &
\end{tabular}

Cuadro 1. Nivel de Autorregulación de los Aprendizaje por Unidad de Formación. Cuestionario ARATEX-R

En relación con la planificación como factor de la autorregulación del aprendizaje, el Cuadro 2 muestra que el $20.9 \%$ presentan niveles: medio alto y alto. Observando habilidades de establecimiento de objetivos y estructuración de un plan de acción para alcanzar los resultados de aprendizaje y la adquisición de conocimiento en las diferentes cátedras en su proceso de formación. El $32.7 \%$ de los participantes presentan nivel medio en el factor planificación de la variable estudiada. El 46.3 \% se agrupan entre los niveles medio bajo y bajo, evidenciando que se cuentan limitadas destrezas de organización de las secuencias de acciones y procedimientos que les permitan alcanzar objetivos de aprendizaje específicos. 


\begin{tabular}{lcc}
\hline NIVEL & fi & hi \% \\
\hline Bajo & 492 & 31.2 \\
Medio bajo & 238 & 15.1 \\
Medio & 516 & 32.7 \\
Medio alto & 169 & 10.7 \\
Alto & 161 & 10.2 \\
Total & $\mathbf{1 5 7 6}$ & $\mathbf{1 0 0 . 0}$ \\
\hline
\end{tabular}

Cuadro 2. Nivel de Planificación. Cuestionario ARATEX-R

Sobre la gestión de la cognición, se pudo encontrar como señala el Cuadro 3 que el $28.3 \%$ de los estudiantes obtienen niveles entre medio alto y alto. Donde se observa el uso de estrategias de repetición, elaboración y organización del a información para alcanzar aprendizajes. El $36.4 \%$ se ubican entre los niveles de bajo y medio bajo. Lo cual permite inferir deficiencias en el uso de estrategias cognitivas, de relacionamiento entre el conocimiento nuevo con el previo. Así como la metacognición orientando de manera consciente la toma de decisiones en sus procesos de estudio con el fin de codificar, almacenar, relacionar y evocar información.

\begin{tabular}{lcc}
\hline NIVEL & fi & hi \% \\
\hline Bajo & 402 & 25.5 \\
Medio bajo & 171 & 10.9 \\
Medio & 558 & 35.4 \\
Medio alto & 129 & 8.2 \\
Alto & 316 & 20.1 \\
Total & $\mathbf{1 5 7 6}$ & $\mathbf{1 0 0 . 0}$ \\
\hline
\end{tabular}

Cuadro 3. Nivel de Gestión de la cognición. Cuestionario ARATEX-R

En referencia a la gestión de la motivación, los hallazgos presentados en el Cuadro 4 indican que el $21.6 \%$ de los estudiantes presentan niveles entre medio alto y alto. Mostrando interés, expectativas y satisfacción tanto en el aprendizaje por sí mismo como en los proceso y productos académicos. El $53.2 \%$ se encuentran entre los niveles: bajo y medio bajo demostrando deficiencia en su motivación tanto intrínseca como extrínseca.

\begin{tabular}{lcc}
\hline NIVEL & fi & hi \% \\
\hline Bajo & 499 & 31.7 \\
Medio bajo & 339 & 21.5 \\
Medio & 398 & 25.3 \\
Medio alto & 181 & 11.5 \\
Alto & 159 & 10.1 \\
Total & $\mathbf{1 5 7 6}$ & $\mathbf{1 0 0 . 0}$ \\
\hline
\end{tabular}

Cuadro 4. Nivel de Gestión de la motivación. Cuestionario ARATEX-R

Sobre la evaluación de la comprensión, en los hallazgos mostrados en el Cuadro 5 se puede observar que al sumar los niveles: alto y medio alto se obtiene que el $25.4 \%$ de los estudiantes pueden ser considerados autorregulados en esta dimensión. Sin embargo, sumando los porcentajes de los niveles: bajo y medio bajo se obtiene el $51 \%$. Más de la 
mitad de los estudiantes encuestados presentan deficiencias en los procesos de autoevaluación de las tareas ejecutadas al momento de analizar textos, lo cual limita la toma de decisiones que permitan conseguir la comprensión de estos.

\begin{tabular}{lcc}
\hline NIVEL & $\mathbf{f i}$ & Hi \\
\hline Bajo & 549 & 34.8 \\
Medio bajo & 256 & 16.2 \\
Medio & 371 & 23.5 \\
Medio alto & 187 & 11.9 \\
Alto & 213 & 13.5 \\
Total & $\mathbf{1 5 7 6}$ & $\mathbf{1 0 0 . 0}$ \\
\hline
\end{tabular}

Cuadro 5. Nivel de Evaluación de la comprensión. Cuestionario ARATEX-R

En relación con la gestión del contexto, se observa en el Cuadro 6 que el $45.7 \%$ de estudiantes encuestados presentan un nivel bajo. Lo cual permite concluir que no planifican las acciones en relación con el contexto antes de analizar un texto. El $25.3 \%$ cuentan con un nivel medio lo que significa que sus destrezas para identificar y controlar los factores del entorno que pueden intervenir en la ejecución de la tarea están en proceso de desarrollo. El $28.9 \%$ se ubican en nivel alto, es decir que utilizan de manera eficiente estrategias de gestión de los recursos individuales y del contexto al momento de analizar un escrito.

\begin{tabular}{lcc}
\hline NIVEL & fi & hi \% \\
\hline Bajo & 721 & 45.7 \\
Medio & 399 & 25.3 \\
Alto & 456 & 28.9 \\
Total & $\mathbf{1 5 7 6}$ & $\mathbf{1 0 0 . 0}$ \\
\hline
\end{tabular}

Cuadro 6. Nivel de Gestión del contexto. Cuestionario ARATEX-R

\section{Discusión de resultados}

Según los resultados obtenidos se establece que la mayoría de los estudiantes poseen un nivel medio bajo y bajo de autorregulación del aprendizaje. Hecho que evidencia sus falencias en actividades de planificación, monitorización y evaluación de sus procesos de formación y consecución de metas académicas. Resultados que concuerdan con los hallazgos de Hernández y Camargo, (2017) quienes establecen que "solamente una cuarta parte de los participantes (24.9\%) presentaron niveles altos en la prueba global.” (p. 14). No obstante, estos resultados difieren de lo encontrado por Gravini, Ortiz y Campo (2016) quienes mencionan que "los resultados generales indican que los estudiantes en su mayoría poseen niveles por encima de la media en sus estrategias metacognitivas, aunque se encuentran algunas diferencias por programas." (p. 326). Lo que evidencia un buen nivel de autorregulación, un adecuado proceso activo y constructivo en los estudiantes para establecer metas en su aprendizaje. Mediante la supervisión, regulación y control de su cognición, motivación y comportamientos.

En relación con los niveles de autorregulación del aprendizaje por sexo no existe diferencia significativa en los resultados obtenidos entre estudiantes hombres y mujeres. Hecho que concuerda con el estudio realizado por Chan y León, (2017) quienes en términos generales mencionan que "no es el género ni el idioma de los aprendices; lo que conducen al desarrollo de un nivel de autorregulación de aprendizaje" (p. 109). No obstante, estos resultados 
contradicen lo encontrado por Ozan, et al. quienes encontraron diferencias significativas entre estudiantes de sexo femenino y masculino a nivel general en las habilidades de autorregulación (Ozan, et al. 2012, p. 1810).

En relación con la autorregulación del aprendizaje por unidad de formación, no se evidencian diferencias significativas, presentando la mayoría de los estudiantes niveles: bajos y medio bajos. Resultados que concuerdan con lo planteado por Hendrie y Bastacini, (2020) quienes mencionan que: "no hay diferencias estadísticamente significativas en general en los puntajes promedios de las estrategias de aprendizaje (evaluadas con el LASSI) en función de la edad" (p. 8).

Sobre la planificación como factor de la autorregulación del aprendizaje, se evidencia que la mayor cantidad de estudiantes no posee destrezas relacionadas a la organización de actividades. Resultados que coinciden con Escorcia, (2010) que manifiesta que "en menor frecuencia, los estudiantes evocan objetivos con relación a la planeación (27\%) y a la revisión (18\%) de los escritos" (p. 271).

Sobre la gestión de la cognición, se halló que los estudiantes tienen deficiencias en el uso de estrategias cognitivas y metacognitivas. Resultados que contradicen lo expuesto por Ozan, et al. (2012) en su investigación. En donde el estudio arrojó que "las percepciones de los estudiantes universitarios sobre la autorregulación metacognitiva, las habilidades de gestión del tiempo y el entorno de estudio, y la autoeficacia se encuentran en un nivel medio" (Ozan, et al. 2012, p. 1810).

En referencia a la gestión de la motivación, los hallazgos obtenidos indican que los estudiantes demuestran niveles: bajo y medio bajo en su motivación intrínseca como extrínseca. Estos resultados contradicen los presentados por Gravini, Ortiz y Campo (2016) en su estudio. En donde "se encontró que todos los estudiantes de los programas evaluados obtuvieron resultados en un nivel alto tanto en la motivación intrínseca como extrínseca" (p. 336).

Sobre la evaluación de la comprensión, los hallazgos señalan que sumando los porcentajes de los niveles: bajo y medio bajo se obtiene que la mitad de los estudiantes encuestados presentan deficiencias en la comprensión al momento de analizar textos. Estos resultados coinciden con los presentados por Pardo (2015). Donde refiere que antes, durante y después de la lectura los estudiantes no establecen los procesos adecuados de exploración, inspección, anticipación y evaluación de su proceso lector. En este sentido, si el estudiante no usa este tipo de estrategias, no se determinará la efectividad del proceso de comprensión y aprendizaje a través de la lectura (Pardo, 2015, pp. 33-36).

En el factor de gestión del contexto, los resultados evidencian que la mayoría de los estudiantes encuestados no planifican las acciones, no monitorizan, ni reflexionan sobre la lectura de un texto. Hallazgos que apoyan lo encontrado por Gravini, Ortiz y Campo (2016) quienes señalan que "todos los programas evaluados demostraron un nivel medio, lo cual significa que solo en algunas ocasiones programan y planean los momentos de estudio determinando específicamente un lugar de trabajo." (p. 339).

\section{Conclusiones}

Los estudiantes de la Facultad de Filosofía, Letras y Ciencias de la Educación de la Universidad Central del Ecuador presentan diferentes niveles de autorregulación del aprendizaje en general, hallando que una mayoría se ubican en los niveles: bajo y medio bajo. Esto permite determinar que los estudiantes presentan falencias en los procesos de 
planificación, control y autoevaluación de sus estrategias de aprendizajes para alcanzar sus metas académicas.

Se encontró que no existen diferencias importantes en los niveles de autorregulación del aprendizaje por sexo. Los estudiantes tanto hombres como mujeres en su mayoría presentan niveles: bajos y medios bajos de la variable estudiada. Los resultados por sexo coinciden también en los otros niveles. Se halló coherencia con los niveles generales, evidenciando falta de habilidades en los estudiantes para establecer objetivos de aprendizaje, ejecutar acciones y monitorear resultados académicos.

No se halló diferencias en los niveles de autorregulación por unidad de formación. El mayor número de estudiantes tanto de la unidad básica, la unidad profesional como de la unidad de titulación, presentan niveles bajos y medio bajos. Estos resultados muestran que la autorregulación, no se adquiere de manera espontánea a lo largo de la formación profesional. Es por tanto indispensable implementar programas educativos que promuevan el desarrollo de estrategias de autorregulación del aprendizaje.

En el factor planificación haciendo un ejercicio de integración con los puntajes: bajo y medio bajo se halló que la mayoría de los estudiantes se ubican en este segmento. Esto evidencia que cuentan no con una aceptable capacidad de anticipar sus acciones y ceñirlas a una programación que les permitan conseguir sus fines de formación. No logrando establecer objetivos que guíen la ejecución de actuaciones eficaces para el autoaprendizaje.

La mayor parte de estudiantes se ubican en niveles: bajos y medio bajos, en el uso de estrategias cognitivas y metacognitivas en sus procesos de estudio y en la consecución del aprendizaje. Presentando una limitada capacidad de relacionar contenidos, utilizar estrategias de organización de información y el poco uso de técnicas de construcción de conocimientos.

De los factores de la autorregulación del aprendizaje estudiados, el que presenta más deficiencias en los participantes es la gestión de la motivación. Donde la mayoría claramente tienen niveles: bajos y medio bajos. Los estudiantes carecen de disposición para hacer esfuerzos extras en los procesos de aprendizaje promovidos tanto por estímulos internos como por gratificaciones. No encuentran interés en guiar sus actos orientados a metas internas o a beneficios externos.

Se encontró que la mayoría de los estudiantes cuentan con niveles: bajos y medio bajos en el uso de estrategias de autoevaluación y evaluación de la comprensión de textos. Esto señala las dificultades que presentan los participantes en observar críticamente su propio desempeño y comprobar cuanto han comprendido de una lectura. Lo cual se replica en las limitaciones en identificar y analizar el resultado de las estrategias usadas para el aprendizaje.

La mayoría de los estudiantes presentan niveles: bajos y medio bajos en la gestión del contexto como factor de la autorregulación del aprendizaje. Los hallazgos señalan que los estudiantes no organizan el ambiente, el tiempo, los recursos o los apoyos que les serian significativos en los logros de aprendizaje.

Tras la contrastación de estos resultados con los revisados en estudios similares se encontró que existen importantes coincidencias, así como resultados contradictorios. Tanto en el nivel general, distribución por sexo, nivel de formación, así como en cada uno de los factores de la autorregulación del aprendizaje. 
En base a estos hallazgos resulta necesario generar innovaciones en el proceso de formación de los estudiantes universitarios. Incluyendo en el currículo programas tendientes al desarrollo de la autorregulación del aprendizaje. Contribuyendo así al logro de sus objetivos académicos y profesionales. También se sugiere profundizar la relación entre esta variable y otras que participan en el proceso de aprendizaje. Por ejemplo, la asociación entre los estilos de enseñanzas de los docentes de esta unidad académica y el nivel de autorregulación del aprendizaje en los estudiantes.

\section{Agradecimientos}

Los Autores agradecen a la Coordinación de Investigación Formativa (CIF) y proyectos semilla de la Universidad Central del Ecuador quienes apoyaron el desarrollo de esta investigación, así como a las autoridades y docentes de la Facultad de Filosofía, Letras y Ciencias de la Educación de le UCE quienes facilitaron y participaron en este estudio.

Presentamos un especial agradecimiento a las señoritas: Alvarado León Michelle Katherine, Quisi Quishpi Paula Johana, León Cabezas Katherine Lizeth, y al señor: Zurita Pacheco Ariel Omar, estudiantes de la Carrera de Psicopedagogía de la UCE quienes fueron miembros del equipo investigador que desarrolló este estudio, por su destacado trabajo y colaboración. 


\section{Bibliografía}

Bandura, A., Barbaranelli, C., Caprara, G. V., y Pastorelli, C. (2001). Self-efficacy beliefs as shapers of children's aspirations and careertrajectories. Child Development, 72 (1), 187-206. https://www.uky.edu/ eushe2/Bandura/Bandura2001CD.pdf

Biggs, J. (1985). The role of meta-learning in study processes. British Journal of Educational Psychology, 55

(1),185-212. https://www.researchgate.net/publication/229763155 The Role of Metalearnin g in Study Processes

Boekaerts, M., y Corno, L. (2005). Self-regulation in the classroom: a perspective on assessment and intervention. Applied psychology: an international review, 54(2), 100-231.

http://sohs.pbs.uam.es/webjesus/motiv ev autorr/lects\%20extranjeras/self\%20 regulation.pdf

Biggs, J. (1993). What do inventories of students' learning processes really measure? A theoretical review and clarification. British Journal of Educational Psychology, 63, 319.

https://www.researchgate.net/publication/14732145 What Do Inventories of St udents' Learning Processes Really Measure A Theoretical Review and Clarificati on

Catuara, S. (2018) Las neuronas espejo. Aprendizaje, imitación y empatía. Ed. Bonalletra Alcompas, S.L.

Castro Pereira. M. (1989), El adulto y su aprendizaje: referencias teóricas y caracterización para el aprendizaje en el contexto de la andragogía, Informe General y Conclusiones de la IX Conferencia Regional Interamericana de Andragogía. Lima, 89-118.

Chan, E., \& León, E. (2017). Exploración del proceso de aprendizaje autorregulado de estudiantes universitarios mayahablantes. Investigación educativa de la REDIECH, 8(1)91-110.

https://www.rediech.org/ojs/2017/index.php/ie rie rediech/article/view/37/32

Cruz, M., Cortés, P., Álvarez, N. (2017). El modelo de Autorregulación y el Aprendizaje Matemático. Congreso Nacional de Investigación Educativa, San Luis Potosí, México, https://www.comie.org.mx/congreso/memoriaelectronica/v14/doc/2072.pdf

Escorcia, D. (2010). Conocimientos metacognitivos y autorregulación: una lectura cualitativa del funcionamiento de los estudiantes universitarios en la producción de textos. Avances En Psicología Latinoamericana,28(2),265-277. https://revistas.urosario.edu.co/index.php/apl/article/view/1456

Gaeta-González, M (2006) Estrategias de autorregulación del aprendizaje: contribución de la orientación de meta y la estructura de metas del aula. Revista Electrónica Interuniversitaria de Formación del Profesorado,9(1),1-8. http://www.aufop.com/aufop/home/

García-Gajardo, F., Fonseca-Grandón, G y Concha-Gfell, L (2015) Aprendizaje y rendimiento académico en educación superior: un estudio comparado. Actualidades Investigativas en

Educación,15(3),1-26. https://www.redalyc.org/pdf/447/44741347019.pdf 
González, A. (2001). Autorregulación del aprendizaje: una difícil tarea. IberPsicología 6 (1),1-25.

https://www.researchgate.net/publication/28059660 Autorregulacion del ap.re ndizaje una dificil tarea/citations

Gravini-Donado, M., Ortiz-Padilla, M. E., \& Campo-Ternera, L. A. (2016). Autorregulación para el aprendizaje en estudiantes universitarios. Educación Y Humanismo, 18(31), 326-342. https://doi.org/10.17081/eduhum.18.31.1382

Hendrie-Kupczyszyn, K y Bastacini, M (2016) Autorregulación en estudiantes universitarios: Estrategias de aprendizaje, motivación y emociones. Revista Scielo, 44 (1), 1-18 https://www.scielo.sa.cr/pdf/edu/v44n1/2215-2644-edu-44-01-00327.pdf

Hernández-Barrios, A y Camargo-Uribe, A (2017) Autorregulación del aprendizaje en la educación superior en Iberoamérica: una revisión sistemática. Revista $\begin{array}{llll}\text { Latinoamericana de Psicología, } & \text { 49(1), }\end{array}$ https://psycnet.apa.org/record/2017-54038-008

Hernández-Barrios, A., \& Camargo-Uribe, Á. (2017). Adaptación y validación del Inventario de Estrategias de Autorregulación en estudiantes universitarios. Suma Psicológica, 24(1), 9-16.

https://reader.elsevier.com/reader/sd/pii/S0121438117300048?token=1B252F 329DDB0B6435F5CB19ECED9B3B832FCFB026A72E310B369F7C608347DB69D6 CDDE45D0162736D1D3C695AE9667

Kaufmann D., Schraw G., Lehman S., (2006) Self-Regulated Learning, ResearchGate 10631073,https://www.researchgate.net/publication/229971208 SelfRegulated Learning

López, S. (1992) Autorregulación y desarrollo de capacidades que incrementan la coherencia entre juicio y acción. Comunicación, lenguaje y Educación 4 (1), 111-118. https://doi.org/10.1080/02147033.1992.10821040

Luy-Montejo, C. (2019). El Aprendizaje Basado en Problemas (ABP) en el desarrollo de la inteligencia emocional de estudiantes universitarios. Propósitos y Representaciones, 7(2), 353-383. http://dx.doi.org/10.20511/pyr2019.v7n2.288

Nuñez, J., Amieiro, N., Álvarez, D., García, T., y Dobarro, A. (2015) Escala de Evaluación de la Autorregulación del Aprendizaje a partir de Textos (ARATEX-R). European Journal of Education and Psychology, 8(1), 9-22. https://www.redalyc.org/pdf/1293/129343965002.pdf

Ozan, C., Kerim , G., Bay, E., \& Celkan, H. (2012). Self-regulated learning strategies skills and self-efficacy perceptions. Social and Behavioral Sciences, 46 (2012) 1806-1811 https://www.researchgate.net/publication/271881025 A Study on the Universit y Students' Self-Regulated Learning Strategies Skills and SelfEfficacy Perceptions in Terms of Different Variables

Panadero, E. \& Alonso-Tapia, J. (2014) ¿Cómo autorregulan nuestros alumnos? Revisión del modelo cíclico de Zimmerman sobre autorregulación del aprendizaje. Anales de $\begin{array}{llll}\text { psicología, } & 30 & \text { (2), 450-462. }\end{array}$ https://www.researchgate.net/publication/260684356 Como autorregulan nuest ros alumnos Modelo de Zimmerman sobre estrategias de aprendizaje/reference $\underline{s}$ 
Pardo, N. (2015). Estrategias autorreguladoras para la comprensión de textos académicos en los estudiantes universitarios. Horizontes Pedagógicos, 17(2), 29-38. https://horizontespedagogicos.ibero.edu.co/article/view/17203/765

Prados, M., Sánchez, V., Sánchez-Queija, I., Del Rey, R., Pertegal, M., Reina, M., Ridao, P., Ortega, F., Mora, J (2014) Manual de Psicología de la Educación. Pirámide https://tecnicasdeaprendizajeefpem.files.wordpress.com/2018/01/manual-depsicologia-de-la-educ.pdf

Pintrich, P. R. (2000). The role of goal orientation in self-regulated learning. En M. Boekaerts, P. Pintrich, y M. Zeidner. Handbook of selfregulation. Academic Press, 452-502.

https://ssrlsite.files.wordpress.com/2018/01/pintrich-2005-the-role-of-goalorientation-in-srl.pdf

Rosário, P., González-Pienda, J. A., Pinto, R., Ferreira, P., Lourenço, A., y Paiva, O. (2010). Efficacy of the program "Testas's (mis)adventures" to promote the deep approach to learning. Psicothema, 22, 828-834. http://www.psicothema.com/pdf/3808.pdf

Rosário, P., Pereira, A., Högemann, J., Nunes, A. R., Figueiredo, M., Núñez, J. C., Fuentes, S., Gaeta, M.L. (2014). Autorregulación del aprendizaje: una revisión sistemática en revistas de la base SciELO. Universitas Psychologica, 13(2), 781-798. https: / $/$ www.redalyc.org/articulo.oa?id=64732221031

Zimmerman, B. J. (2001). Theories of self-regulated learning and academic achivement: An overview and analysis. B. J. Zimmerman \& D. H. Schunk. Self-regulated learning and academic achievement: Theoretical perspectives, Lawrence Erlbaum Associates Publishers. (2) 1-38.

Zimmerman, B.J. \& Martinez-Pons, M. (1998). Construct validation of a strategy model of student self-regulated learning. Journal of Educational Psychology, 80(3), 284-290.

Zimmerman, B. J., \& Schunk, D. H. (2011). Self-regulated learning and performance: An introduction and an overview. B. J. Zimmerman \& D. H. Schunk. Handbook of selfregulation of learning and performance. Routledge. 1-12.

Zimmerman, B. J. (2008). Investigating self-regulation and motivation: Historical, background, methodological developments, and future prospects. American Educational Research Journal, 45, 166-183. https://www.researchgate.net/publication/250184865 Investigating SelfRegulation and Motivation Historical Background Methodological Developments and Future Prospects 


\section{Autores}

PABLO BURBANO-LARREA, Obtuvo el título de Magister en Neuropsicología Educativa en la Universidad Internacional de la Rioja en el 2020. Obtuvo el título de Magister en Ciencias de la Educación en la Pontificio Universidad Católica del Ecuador en el 2015. Obtuvo el título de Psicólogo Clínico en la Universidad Central del Ecuador en el 2010.

Actualmente es Docente a nivel superior en la Universidad Central del Ecuador. También Trabajo como docente en la Pontificia Universidad Católica del Ecuador. Se ha desempeñado en cargos directivos en Instituciones de Educación Básica, Media y Superior. Cuenta con formación complementaria en investigación, pedagogía, didáctica y psicología. Ha realizado ponencias en eventos nacionales e Internacionales. Se ha desempeñado como Orientador familiar y matrimonial, así como Terapeuta en ejercicio profesional. Tutor de proyectos de investigación de grado. Ha publicado sobre violencia de género en la Revista de Ciencias Sociales de la Universidad del Zuliay (En revisión). Capitulo Estrategias para enfrentar la educación virtual en tiempos de Covid-19 del Libro Coalición por la Educación.

MIRIAN BASANTES-VÁSQUEZ obtuvo su título de Magíster en Gerencia de Proyectos Educativos y Sociales, por la Facultad de Filosofía, Letras y Ciencias de la Educación de la Universidad Central del Ecuador (Ecuador) en 2004. Obtuvo su título de Magíster en Educación Sexual, de la Universidad Técnica del Norte (Ecuador) en 2004. Obtuvo su título de Doctora en Psicología Educativa y Orientación de la Facultad de Filosofía, Letras y Ciencias de la Educación de la Universidad Central del Ecuador (Ecuador) en 1984. Obtuvo su título de Licenciada en Ciencias de la Educación, profesora de segunda enseñanza, especialización Psicología Educativa y Orientación Vocacional por la Facultad de Filosofía, Letras y Ciencias de la Educación de la Universidad Central del Ecuador (Ecuador) en 1980.

Actualmente es profesora titular de la Facultad de Filosofía, Letras y Ciencias de la Educación de la Universidad Central del Ecuador en diferentes áreas de Psicología y Educación. Sus principales temas de investigación incluyen la psicología del desarrollo, psicología del aprendizaje, sexualidad, maltrato infantil, dificultades de aprendizaje, psicología social, orientación educativa. Es autora de libros y capítulos de libros relacionados a los temas de investigación. Es tutora de trabajos de investigación de grado de la Facultad de Filosofía, Letras y Ciencias de la Educación de la Universidad Central del Ecuador y tutora de proyectos de posgrado de la Universidad Tecnológica Indoamérica.

ISABEL RUIZ-LAPUERTA, obtuvo el título de Máster en Psicopedagogía en la Universidad Internacional de la Rioja en el 2020, obtuvo el título de Magister en Neuropsicología Infantil en el año 2012 en la Universidad Central del Ecuador, obtuvo el título de Doctora en Psicología Clínica en el año 2002 en la Universidad Central del Ecuador. Ha desempeñado actividades profesionales en instituciones educativas, centros psicopedagógicos, centro de adolescentes infractores y consulta privada.

Actualmente es Docente titular de la Facultad de Filosofía, Letras y Ciencias de la Educación, en las carreras de Psicología Educativa y Psicopedagogía; es tutora de proyectos de investigación de grado en la carrera de Psicología Educativa y Orientación. 